
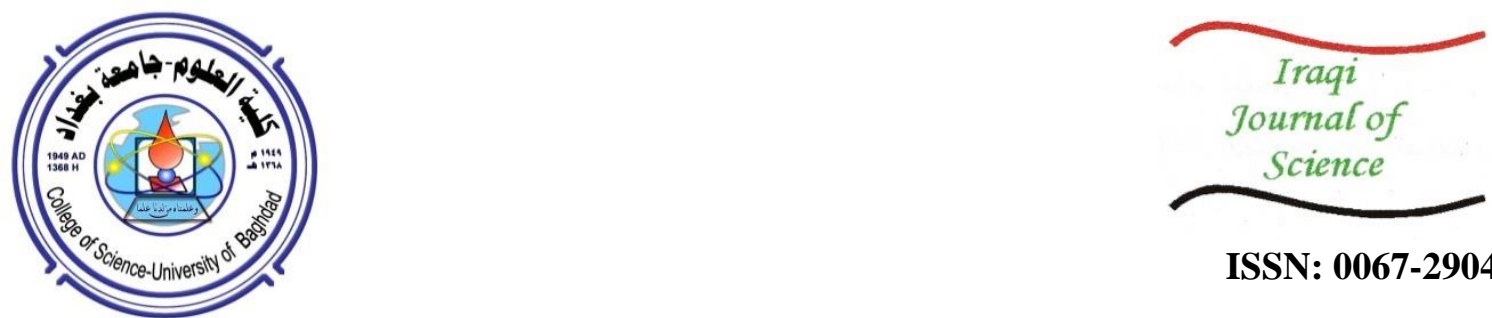

ISSN: 0067-2904

\title{
Simplified Flat Coordinate Model for Northern Parts of Arabian Gulf
}

\author{
Ali Abdulrazzaq Saud*1, Loay E. George ${ }^{2}$, Baqer H. Sayyid ${ }^{3}$ \\ ${ }^{1}$ University of Baghdad, College of Science, Physics Department \\ ${ }^{2}$ University of Information Technology and Communication \\ ${ }^{3}$ University of Baghdad, College of Science, Remote Sensing \& GIS Department
}

Received: 10/10/2020

Accepted: 16/3/2021

\begin{abstract}
The method of coordinate conversion is still considered important and laborious due to the shift from the spatial ellipsoidal (geographic) to the flat planned system. The most common method uses a contiguous UTM system as one of the most reliable systems in the conversion process; however, this system faces a problem in large areas that contain more than one zone. The goal of this research is to create a simple and low computational cost model to represent a non-contiguous semi-UTM geographic coordinates for confined regions of the globe. The considered region taken in this study is the northern parts of Arabian Gulf (including parts of Iraq, Kuwait, Iran, and Saudi Arabia). The determined mathematical model was based on using two dimensional Taylor sequence. The most accurate representation met in this study was based on the $6^{\text {th }}$ two dimensional polynomial. The estimation of equations' coefficients was done using the least square criterion for the overall error of estimating coordinate values of either (latitude, longitude) or (Easting, Northing). The two basic determinations were applied for the forward and the backward; in the first step, the conversion of coordinates was calculated from the ellipsoidal coordinates (i.e., Longitude, Latitude) to UTM (WGS84) coordinates (i.e., Easting, Northing) and vice versa.

The attained results indicated that the mathematical model used is successful for achieving the conversion process. With the use of the $6^{\text {th }}$ order $2 \mathrm{D}$-polynomial equations, a very small error of less than $1 \mathrm{~m}$ was achieved in the Easting and Northing coordinates.
\end{abstract}

Keywords: Geographic Coordinate System, UTM, Polynomial Representation, Taylor Series

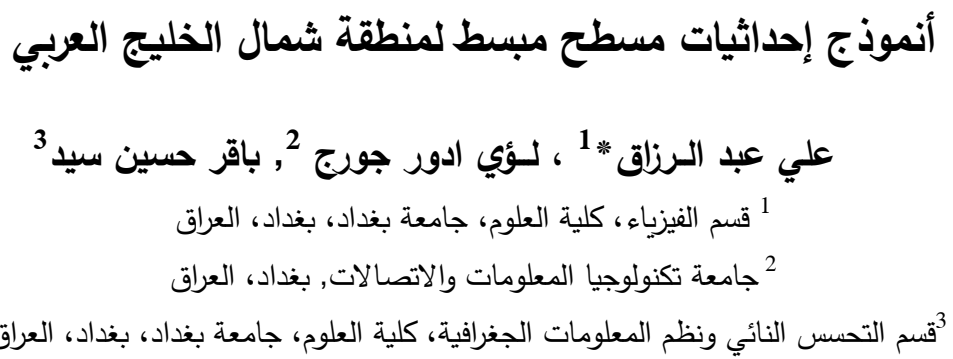

الخلاصة

لا تزال عملية تحويل الإحداثيات من المهام الصعبة بالرغم من وجود طرق مختلفة للتحويل من النظام

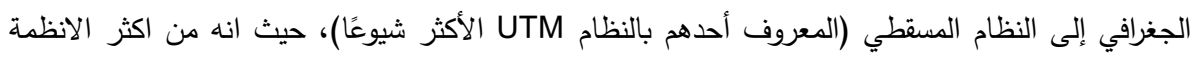

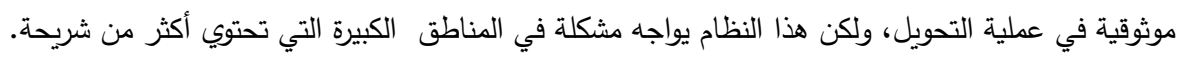

*Email: Aliabd9888@yahoo.com 


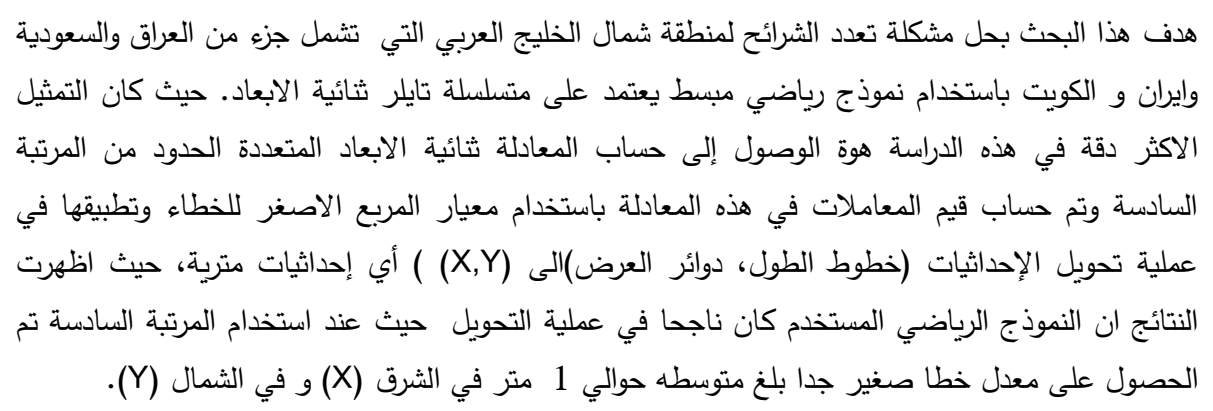

\section{Introduction}

Universal Transverse Mercator (UTM) is an international plane (rectangular) coordinate system developed by the U.S. Army [1]. In this system, the world is divided into 60 zones, each covering 6 degrees of longitude. In latitude, it extends from $80^{\circ} \mathrm{S}$ to $84^{\circ} \mathrm{N}$. The origin of each zone is the intersection of the central meridian at the equator. High degree of accuracy is possible due to separate projections for each UTM zone. UTM values are calculated in meters. To eliminate negative coordinates, the projection alters the coordinate values at the origin. The value given to the central meridian is the false easting and the value assigned to the Equator is the false northing. For locations in the Northern hemisphere, the origin is assigned a false easting of 500,000 meters and a false northing of 0 . For locations in the southern hemisphere, the origin is assigned a false easting of 500,000 meters and a false northing of 10,000,000 meters $(10,000 \mathrm{~km})[2,3,4]$. The surface of the Earth cannot be flatten unless it is converted to a plane form . The UTM can provide a method to represent every point on earth by using a list of flat (X, Y or Easting, Northing) coordinates [5]. The benefits of using the flat coordinates for representing the location on earth is for easy planar mapping and easy derivation of spatial information from locations coordinates (e.g., distances, angles, areas, etc.), added to that the capability to determine the location of a point is dependent on spatial information. The measurement of distances, directions, and areas can be performed more efficiently than the geographic coordinate system, due the shifting of the actual location in the geographic coordinate [6]. There is a problem when converting coordinates in UTM system, but it is a small problem when compared with other systems. The principle of UTM system is to divide the world into zones, where each area within the zone has its own special attributes. Most of the areas are within more than one zone; therefore, moving from one zone to another makes the converting result inaccurate, which in fact is the root of the problem [7, 8]. In Iraq, the same problem exists in the UTM Extended Zones, as the territory of Iraq falls within the UTM system in three regions (37, 38 and 39). Zone 37 includes parts of western Iraq, zone 38 covers the central region and includes most of Iraq, and zone 39 covers a small part of the city of Basra in eastern Iraq [9]. The same problem happens with the north parts of the Arabian Gulf, where the area of the gulf lies in three different zones, which are $(38,39,40)$; zone 38 covers parts of Basra, Kuwait and Saudi Arabia, zone 39 covers Bahrain, Qatar and United Arab Emirates, and zone 40 covers parts of Iran. Figure-1 illustrates the coverage area of zones 38, 39 and 40 in the northern parts of Arabian Gulf.

\section{Theoretical Framework}

In this section, the mathematical framework of the transfer is explained. Four models of polynomial equations were used to perform the transformation.

\section{Absolute Polynomial Representation}

$$
\begin{array}{llrl}
\text { Forward: } & X_{k}=\sum_{i=0}^{\text {ord }} \sum_{j=0}^{i} a_{L} \lambda_{k}^{j} \varphi_{k}^{i-j} ; & Y_{k}=\sum_{i=0}^{\text {ord }} \sum_{j=0}^{i} b_{L} \lambda_{k}^{j} \varphi_{k}^{i-j} \\
\text { Inverse: } & \lambda_{k}=\sum_{i=0}^{\text {ord }} \sum_{j=0}^{i} c_{L} X_{k}^{j} Y_{k}^{i-j} ; & \varphi=\sum_{i=0}^{\text {ord }} \sum_{j=0}^{i} d_{L} X_{k}^{j} Y_{k}^{i-j}
\end{array}
$$

\section{Relative Polynomial Representation:}

$$
\text { Forward: } \quad X_{k}=\sum_{i=0}^{\text {ord }} \sum_{j=0}^{i} a_{L}\left(\lambda_{k}-\bar{\lambda}\right)^{j}\left(\varphi_{k}-\bar{\varphi}\right)^{i-j}
$$




$$
\begin{aligned}
Y_{k} & =\sum_{i=0}^{\text {ord }} \sum_{j=0}^{i} b_{L}\left(\lambda_{k}-\bar{\lambda}\right)^{j}\left(\varphi_{k}-\bar{\varphi}\right)^{i-j} \\
\text { Inverse: } \quad \lambda_{k} & =\sum_{i=0}^{\text {ord }} \sum_{j=0}^{i} c_{L}\left(X_{k}-\bar{X}\right)^{j}\left(Y_{k}-\bar{Y}\right)^{i-j} \\
\varphi_{k} & =\sum_{i=0}^{\text {ord }} \sum_{j=0}^{i} d_{L}\left(X_{k}-\bar{X}\right)^{j}\left(Y_{k}-\bar{Y}\right)^{i-j}
\end{aligned}
$$

\section{Normalized Polynomial Representation}

$$
\begin{array}{ll}
\text { Forward: } & X_{i}=\sum_{i=0}^{\text {Ord }} \sum_{j=0}^{i} a_{L}\left(\frac{\lambda_{i}}{\bar{\lambda}}\right)^{j}\left(\frac{\varphi_{i}}{\bar{\varphi}}\right)^{i-j} ; \quad Y_{i}=\sum_{i=0}^{\text {Ord }} \sum_{j=0}^{i} b_{L}\left(\frac{\lambda_{i}}{\bar{\lambda}}\right)^{j}\left(\frac{\varphi_{i}}{\bar{\varphi}}\right)^{i-j} \\
\text { Inverse: } & \lambda_{k}=\sum_{i=0}^{\text {Ord }} \sum_{j=0}^{i} c_{L}\left(\frac{X_{k}}{\bar{X}}\right)^{j}\left(\frac{Y_{k}}{\bar{Y}}\right)^{i-j} ; \quad \varphi_{k}=\sum_{i=0}^{\text {Ord }} \sum_{j=0}^{i} d_{L}\left(\frac{X_{k}}{\bar{X}}\right)^{j}\left(\frac{Y_{k}}{\bar{Y}}\right)^{i-j}
\end{array}
$$

\section{Relative Normalized Polynomial Representation}

$$
\begin{aligned}
& \text { Forward: } \quad X_{k}=\sum_{i=0}^{\text {ord }} \sum_{j=0}^{i} a_{L}\left(\frac{\lambda_{k}-\bar{\lambda}}{\bar{\lambda}}\right)^{j}\left(\frac{\varphi_{k}-\bar{\varphi}}{\bar{\varphi}}\right)^{i-j} \\
& Y_{k}=\sum_{i=0}^{\text {ord }} \sum_{j=0}^{i} b_{L}\left(\frac{\lambda_{k}-\bar{\lambda}}{\bar{\lambda}}\right)^{j}\left(\frac{\varphi_{k}-\bar{\varphi}}{\bar{\varphi}}\right)^{i-j} \\
& \text { Inverse: } \quad \lambda_{k}=\sum_{i=0}^{\text {ord }} \sum_{j=0}^{i} c_{L}\left(\frac{X_{k}-\bar{X}}{\bar{X}}\right)^{j}\left(\frac{Y_{k}-\bar{Y}}{\bar{Y}}\right)^{i-j} \\
& \varphi_{k}=\sum_{i=0}^{\text {ord }} \sum_{j=0}^{i} d_{L}\left(\frac{X_{k}-\bar{X}}{\bar{X}}\right)^{j}\left(\frac{Y_{k}-\bar{Y}}{\bar{Y}}\right)^{i-j}
\end{aligned}
$$

where $\lambda_{k}, \varphi_{k}, X_{k}$, and $Y_{k}$ are the latitude and longitude (East, North) coordinates, respectively, of $k^{\text {th }}$ point, and the corresponding mean values are:

$$
\begin{array}{cl}
\bar{\lambda}=\frac{1}{N} \sum_{k=1}^{N} \lambda_{k}, & \bar{\varphi}=\frac{1}{N} \sum_{k=1}^{N} \varphi_{k} \\
\bar{X}=\frac{1}{N} \sum_{k=1}^{N} X_{k}, & \bar{Y}=\frac{1}{N} \sum_{k=1}^{N} Y_{k}
\end{array}
$$

where $\left\{a_{L}\right\},\left\{b_{L}\right\},\left\{c_{L}\right\},\left\{d_{L}\right\}$ are the sequence of polynomial coefficients.

After testing the four modes, the attained results show that the Relative Normalized Polynomial is the best to use.

\subsection{Forward Representation}

The coordinate values of East and North (X and $\mathrm{Y}$ ) can be represented mathematically, with changing the symbols of latitude and longitude from $\{\boldsymbol{\varphi}, \lambda\}$ to $\{\alpha, \beta\}$ and the polynomial coefficients from $\{a, b, c, d\}$ to $\{1, \mathrm{~m}, \mathrm{o}, \mathrm{q}\}$, and substituting them in the relative normalized polynomial, as follows:

$$
X=F(\alpha, \beta), \quad Y=G(\alpha, \beta)
$$

These functions are continuous over most of the regions of globe, except the poles. Thus the Taylor series [10] of both functions near the point $\left(\alpha_{o}, \beta_{o}\right)$ can be calculated using the equations (5a) and (5b) above. By applying the $6^{\text {th }}$ order polynomial equation, we get equation (6a) and (6b) as shown below. 


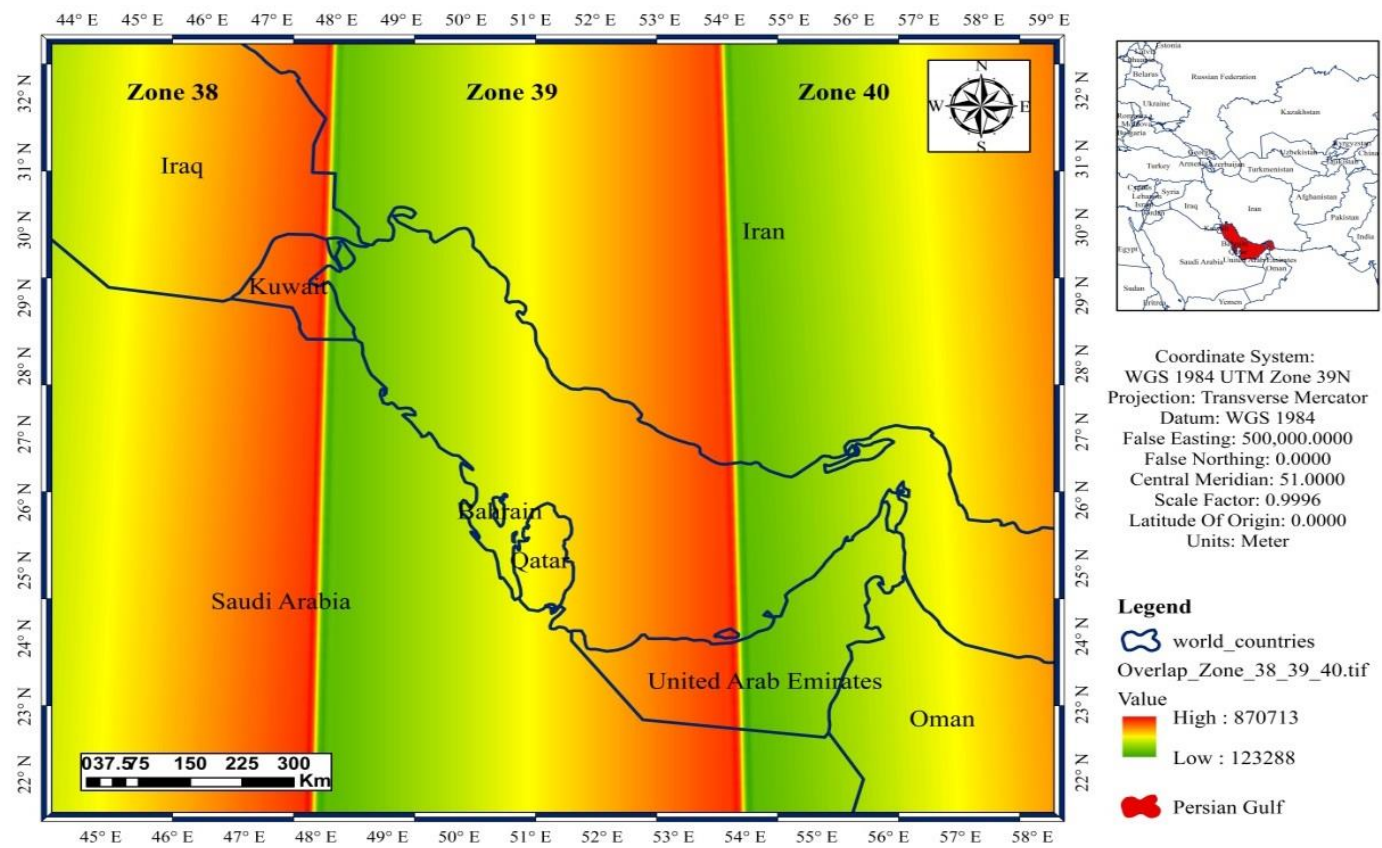

Figure 1-The overlap between the zones 38, 39 and 40

\section{For the $6^{\text {th }}$ Order Polynomials for Absolute Polynomial}

$$
\begin{gathered}
X=l_{0}+l_{1} \Delta \beta+l_{2} \Delta \alpha+l_{3}(\Delta \beta)^{2}+l_{4} \Delta \alpha \Delta \beta+l_{5}(\Delta \alpha)^{2}+l_{6}(\Delta \beta)^{3}+l_{7}(\Delta \beta)^{2} \Delta \alpha \\
+l_{8} \Delta \beta(\Delta \alpha)^{2}+l_{9}(\Delta \alpha)^{3}+l_{10}(\Delta \beta)^{4}+l_{11}(\Delta \beta)^{3} \Delta \alpha+l_{12}(\Delta \beta)^{2}(\Delta \alpha)^{2}+l_{13} \Delta \beta(\Delta \alpha)^{3}+ \\
l_{14}(\Delta \alpha)^{4}+l_{15}(\Delta \beta)^{5}+l_{16}(\Delta \beta)^{4} \Delta \alpha+l_{17}(\Delta \beta)^{3}(\Delta \alpha)^{2}+l_{18}(\Delta \beta)^{2}(\Delta \alpha)^{3}+ \\
l_{19} \Delta \beta(\Delta \alpha)^{4}+l_{20}(\Delta \alpha)^{5}+l_{21}(\Delta \beta)^{6}+l_{22}(\Delta \beta)^{5} \Delta \alpha+l_{23}(\Delta \beta)^{4}(\Delta \alpha)^{2}+ \\
l_{24}(\Delta \beta)^{3}(\Delta \alpha)^{3}+l_{25}(\Delta \beta)^{2}(\Delta \alpha)^{4}+l_{26}(\Delta \beta) \Delta \alpha^{5}+ \\
l_{27} \Delta \alpha \\
Y=m_{0}+m_{1} \Delta \beta+m_{2} \Delta \alpha+m_{3}(\Delta \beta)^{2}+m_{4} \Delta \alpha \Delta \beta+m_{5}(\Delta \alpha)^{2}+m_{6}(\Delta \beta)^{3}+m_{7}(\Delta \beta)^{2} \Delta \alpha \\
\quad+m_{8} \Delta \beta(\Delta \alpha)^{2}+m_{9}(\Delta \alpha)^{3}+m_{10}(\Delta \beta)^{4}+m_{11}(\Delta \beta)^{3} \Delta \alpha+m_{12}(\Delta \beta)^{2}(\Delta \alpha)^{2} \\
+m_{13} \Delta \beta(\Delta \alpha)^{3}+m_{14}(\Delta \alpha)^{4}+m_{15}(\Delta \beta)^{5}+m_{16}(\Delta \beta)^{4} \Delta \alpha+m_{17}(\Delta \beta)^{3}(\Delta \alpha)^{2} \\
+m_{18}(\Delta \beta)^{2}(\Delta \alpha)^{3}+m_{19} \Delta \beta(\Delta \alpha)^{4}+m_{20}(\Delta \alpha)^{5}+m_{21}(\Delta \beta)^{6}+m_{22}(\Delta \beta)^{5} \Delta \alpha \\
+m_{23}(\Delta \beta)^{4}(\Delta \alpha)^{2}+m_{24}(\Delta \beta)^{3}(\Delta \alpha)^{3}+m_{25}(\Delta \beta)^{2}(\Delta \alpha)^{4}+m_{26}(\Delta \beta) \Delta \alpha^{5} \\
\quad+m_{27} \Delta \alpha
\end{gathered}
$$

\subsection{Backward Representation}

Also, the inverse mapping equations can be approximately written in the form:

$$
\alpha=H(X, Y), \beta=Q(X, Y)
$$

Since the above equation is continuous in the region, then the Taylor series $[11,12]$ for both equations $(4 \mathrm{c})$ and $(4 \mathrm{~d})$ can be written as in equations (8a) and (8b):

$$
\begin{aligned}
& \alpha=o_{0}+o_{1} \Delta y+o_{2} \Delta x+o_{3}(\Delta y)^{2}+o_{4} \Delta x \Delta y+o_{5}(\Delta x)^{2}+o_{6}(\Delta y)^{3}+o_{7}(\Delta y)^{2} \Delta x \\
& +o_{8} \Delta y(\Delta x)^{2}+o_{9}(\Delta x)^{3}+o_{10}(\Delta y)^{4}+o_{11}(\Delta y)^{3} \Delta x+o_{12}(\Delta y)^{2}(\Delta x)^{2} \\
& +o_{13} \Delta y(\Delta x)^{3}+o_{14}(\Delta x)^{4}+o_{15}(\Delta y)^{5}+o_{16}(\Delta y)^{4} \Delta x+o_{17}(\Delta y)^{3} \Delta x^{2}+o_{18}(\Delta y)^{2} \Delta x^{3}+ \\
& o_{19} \Delta y \Delta x^{4}+o_{20} \Delta x^{5}+o_{21}(\Delta y)^{6}+o_{22}(\Delta y)^{5} \Delta x o_{23}(\Delta y)^{4} \Delta x^{2}+o_{24}(\Delta y)^{3} \Delta x^{3}+o_{25}(\Delta y)^{2} \Delta x^{4}+ \\
& o_{26}(\Delta y) \Delta x^{5}+o_{27} \Delta x^{6} \\
& \beta=p_{0}+p_{1} \Delta y+p_{2} \Delta x+p_{3}(\Delta y)^{2}+p_{4} \Delta x \Delta y+p_{5}(\Delta x)^{2}+p_{6}(\Delta y)^{3}+p_{7}(\Delta y)^{2} \Delta x \\
& +p_{8} \Delta y(\Delta x)^{2}+p_{9}(\Delta x)^{3}+p_{10}(\Delta y)^{4}+p_{11}(\Delta y)^{3} \Delta x+p_{12}(\Delta y)^{2}(\Delta x)^{2} \\
& +p_{13} \Delta y(\Delta x)^{3}+p_{14}(\Delta x)^{4}+p_{15}(\Delta y)^{5}+p_{16}(\Delta y)^{4} \Delta x+p_{17}(\Delta y)^{3} \Delta x^{2}+p_{18}(\Delta y)^{2} \Delta x^{3}+ \\
& p_{19} \Delta y \Delta x^{4}+p_{20} \Delta x^{5}+p_{21}(\Delta y)^{6}+p_{22}(\Delta y)^{5} \Delta x+p_{23}(\Delta y)^{4} \Delta x^{2}+p_{24}(\Delta y)^{3} \Delta x^{3}+ \\
& p_{25}(\Delta y)^{2} \Delta x^{4}+p_{26}(\Delta y) \Delta x^{5}+p_{27} \Delta x^{6}
\end{aligned}
$$

\section{Results and discussion}

The dataset used in this study represents the grid of points' coordinates (Longitude, Latitude) covering 
the north gulf region with some surrounding areas. The separation $t$ of difference between the grid points is taken as 0.25 degrees. These coordinates were then converted to $(\mathrm{X}, \mathrm{Y})$ coordinates using UTM system conversion software but we will determine this program on Zones 38 and calculate (X, Y) coordinate values, as shown in Figure-2. The tables below show error rates and explain why we selected the relative normalized polynomial over the absolute polynomial, relative polynomial, and normalized polynomial.

Table 1-The error rate values using the $6^{\mathrm{TH}}$ order polynomials equations for absolute polynomial representation

\begin{tabular}{|c|c|c|}
\hline Parameter & Error rate at X easting $(\mathrm{m})$ & Error rate at Y northing $(\mathrm{m})$ \\
\hline Maximum & 18.29775 & 4.904424 \\
\hline Minimum & 0.00748 & 0.000162 \\
\hline Average & 3.166547 & 0.622365 \\
\hline
\end{tabular}

Table 2-The error rate values using the $6^{\mathrm{TH}}$ order polynomials equations for relative polynomial representation

\begin{tabular}{|c|c|c|}
\hline Parameter & Error rate at X easting $(\mathrm{m})$ & Error rate at Y northing $(\mathrm{m})$ \\
\hline Maximum & 424541.6 & 637261.3 \\
\hline Minimum & $4.70995 \mathrm{E}-05$ & 0.047143 \\
\hline Average & 255872.5 & 286367.5 \\
\hline
\end{tabular}

Table 3- The error rate values using the $6^{\mathrm{TH}}$ order polynomials equations for normalized polynomial representation

\begin{tabular}{|c|c|c|}
\hline Parameter & Error rate at X easting $(\mathrm{m})$ & Error rate at Y northing $(\mathrm{m})$ \\
\hline Maximum & 716070.4 & 12402307 \\
\hline Minimum & 187.4763 & 3968.041 \\
\hline Average & 211199.3 & 3628554 \\
\hline
\end{tabular}

The results listed in the error rate tables explain the reason behind selecting the relative normalized polynomial equations in this study.

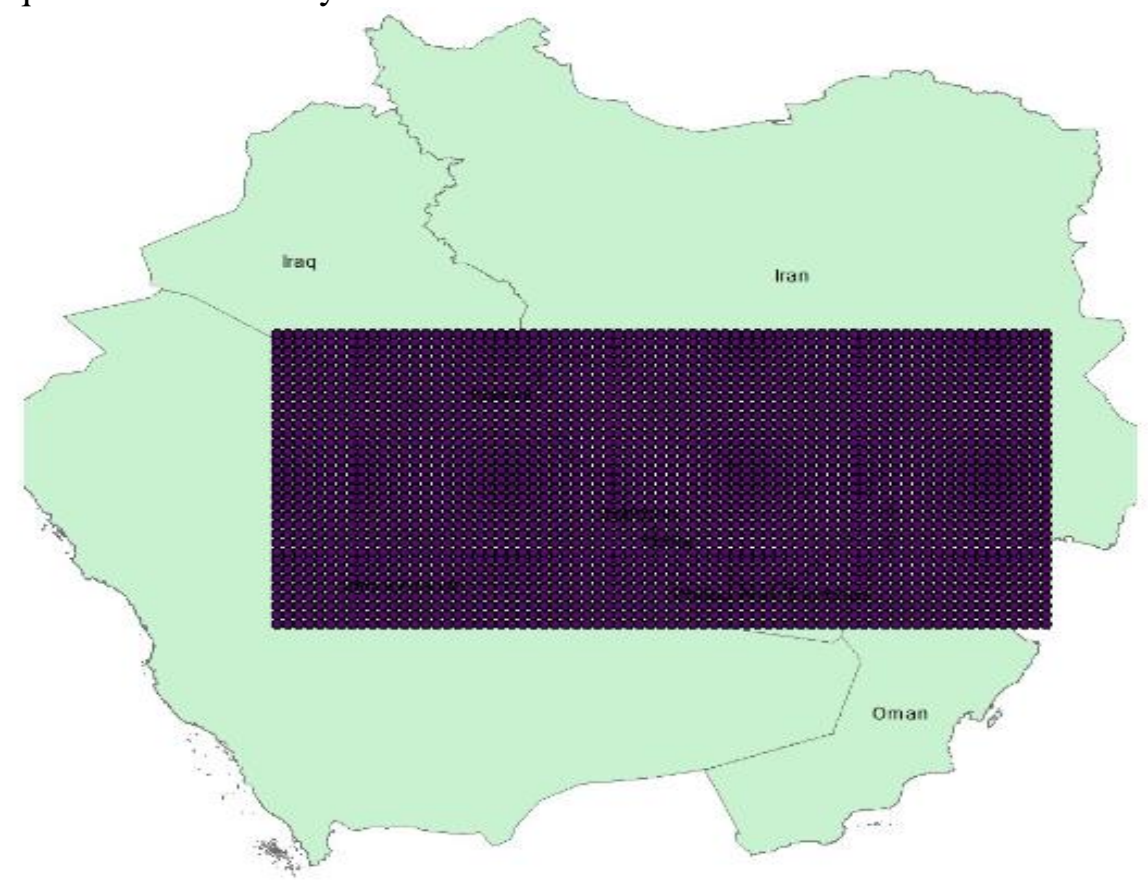

Figure 2-The data set (grids point)

\subsection{Forward}

This step presents the calculation rate of error for test two at $\mathrm{X}$ east and $\mathrm{Y}$ north using different orders polynomial equations (up to $5^{\text {th }}$ order), depending on least square error criteria. The tables below list the error rates for these orders, depending on the relative normalized polynomial equation. 
Table 4-The error rate values using the first order polynomials equations (6a \&6b)

\begin{tabular}{|c|c|c|}
\hline Parameter & Error rate at X easting $(\mathrm{m})$ & Error rate at Y northing $(\mathrm{m})$ \\
\hline Maximum & 52901.68 & 30132.11 \\
\hline Minimum & 7.190227 & 10.12198 \\
\hline Average & 12833.68 & 8697.298 \\
\hline
\end{tabular}

Table 5-The error rate values using the $2^{\text {nd }}$ order polynomials equations ( $6 \mathrm{a} \& 6 \mathrm{~b}$ )

\begin{tabular}{|c|c|c|}
\hline Parameter & Error rate at X easting $(\mathrm{m})$ & Error rate at Y northing $(\mathrm{m})$ \\
\hline Maximum & 4366.735 & 3802.241 \\
\hline Minimum & 0.035138 & 0.196596 \\
\hline Average & 774.6515 & 674.6406 \\
\hline
\end{tabular}

Table 6-The error rate values using the $3^{\text {rd }}$ order polynomials equations ( $6 \mathrm{a} \& 6 \mathrm{~b}$ )

\begin{tabular}{|l|l|l|}
\cline { 2 - 3 } Parameter & Error rate at X easting $(\mathrm{m})$ & Error rate at Y northing $(\mathrm{m})$ \\
\hline Maximum & 681.9945 & 295.3592 \\
\hline Minimum & 0.062986 & 0.038521 \\
\hline Average & 105.1864 & 55.43351 \\
\hline
\end{tabular}

Table 7-The error rate values using the $4^{\text {th }}$ order polynomials equations (7a \& 7b)

\begin{tabular}{|c|c|c|}
\hline Parameter & Error rate at X easting $(\mathrm{m})$ & Error rate at Y northing $(\mathrm{m})$ \\
\hline Maximum & 117.3495 & 17.04874 \\
\hline Minimum & 0.008332 & 0.000311 \\
\hline Average & 16.78372 & 1.875654 \\
\hline
\end{tabular}

Table 8-The error rate values using the $5^{\text {th }}$ order polynomials equations (6a \&6b)

\begin{tabular}{|c|c|c|}
\hline Parameter & Error rate at X easting $(\mathrm{m})$ & Error rate at Y northing $(\mathrm{m})$ \\
\hline Maximum & 16.29331 & 1.460375 \\
\hline Minimum & 0.000674 & 0.000169 \\
\hline Average & 2.691837 & 0.269368 \\
\hline
\end{tabular}

According to the error rate values of the orders above, we concluded that the $6^{\text {th }}$ order gives the minimum error value.

Tables-9 and 10 show the values of polynomial coefficients and values of attained error rates in $\mathrm{X}$ east and $\mathrm{Y}$ north using the $6^{\text {th }}$ order polynomial (equations $6 \mathrm{a}$ and $6 \mathrm{~b}$ ). Also, the error distributions as shown in Figures-(3 and 4)

Table 9-Values of parameters using the $6^{\text {th }}$ order polynomial equations $(6 \mathrm{a} \& 6 \mathrm{~b}$ )

\begin{tabular}{|l|l|l|l|l|l|l|l|}
\hline Symbol & Coefficients & Symbol & Coefficients & Symbol & Coefficients & Symbol & Coefficients \\
\hline $\mathrm{L}_{0}$ & $-3.61 \mathrm{E}-04$ & $\mathrm{~L}_{14}$ & $4.72 \mathrm{E}-02$ & $\mathrm{M}_{0}$ & $-3.68 \mathrm{E}-03$ & $\mathrm{M}_{14}$ & $-8.07 \mathrm{E}-03$ \\
\hline $\mathrm{L}_{1}$ & 4.630459 & $\mathrm{~L}_{15}$ & 0.141462 & $\mathrm{M}_{1}$ & $8.04 \mathrm{E}-02$ & $\mathrm{M}_{15}$ & $1.20 \mathrm{E}-02$ \\
\hline $\mathrm{L}_{2}$ & -0.13066 & $\mathrm{~L}_{16}$ & $-2.47 \mathrm{E}-04$ & $\mathrm{M}_{2}$ & 0.996175192 & $\mathrm{M}_{16}$ & $1.45 \mathrm{E}-05$ \\
\hline $\mathrm{L}_{3}$ & 0.129273 & $\mathrm{~L}_{17}$ & -1.97506 & $\mathrm{M}_{3}$ & 0.34659287 & $\mathrm{M}_{17}$ & $2.24 \mathrm{E}-03$ \\
\hline $\mathrm{L}_{4}$ & $-6.03 \mathrm{E}-02$ & $\mathrm{~L}_{18}$ & 0.146082 & $\mathrm{M}_{4}$ & $-1.95 \mathrm{E}-04$ & $\mathrm{M}_{18}$ & $-4.40 \mathrm{E}-02$ \\
\hline $\mathrm{L}_{5}$ & -1.12852 & $\mathrm{~L}_{19}$ & 0.11841 & $\mathrm{M}_{5}$ & $5.48 \mathrm{E}-02$ & $\mathrm{M}_{19}$ & $-3.32 \mathrm{E}-02$ \\
\hline $\mathrm{L}_{6}$ & 0.376398 & $\mathrm{~L}_{20}$ & $7.01 \mathrm{E}-03$ & $\mathrm{M}_{6}$ & $4.07 \mathrm{E}-02$ & $\mathrm{M}_{20}$ & $2.94 \mathrm{E}-03$ \\
\hline $\mathrm{L}_{7}$ & $4.81 \mathrm{E}-03$ & $\mathrm{~L}_{21}$ & $1.76 \mathrm{E}-06$ & $\mathrm{M}_{7}$ & $-4.01 \mathrm{E}-05$ & $\mathrm{M}_{21}$ & $1.71 \mathrm{E}-02$ \\
\hline $\mathrm{L}_{8}$ & -0.25187 & $\mathrm{~L}_{22}$ & $3.37 \mathrm{E}-05$ & $\mathrm{M}_{8}$ & 0.23335314 & $\mathrm{M}_{22}$ & $-6.23 \mathrm{E}-06$ \\
\hline $\mathrm{L}_{9}$ & -0.51441 & $\mathrm{~L}_{23}$ & -3.3576 & $\mathrm{M}_{9}$ & $-3.60 \mathrm{E}-02$ & $\mathrm{M}_{23}$ & $-1.24 \mathrm{E}-02$ \\
\hline $\mathrm{L}_{10}$ & $6.97 \mathrm{E}-02$ & $\mathrm{~L}_{24}$ & 0.789543 & $\mathrm{M}_{10}$ & $8.89 \mathrm{E}-02$ & $\mathrm{M}_{24}$ & $-9.71 \mathrm{E}-02$ \\
\hline $\mathrm{L}_{11}$ & $1.14 \mathrm{E}-03$ & $\mathrm{~L}_{25}$ & 0.490981 & $\mathrm{M}_{11}$ & $1.59 \mathrm{E}-05$ & $\mathrm{M}_{25}$ & $6.06 \mathrm{E}-03$ \\
\hline $\mathrm{L}_{12}$ & -1.02824 & $\mathrm{~L}_{26}$ & $-2.00 \mathrm{E}-02$ & $\mathrm{M}_{12}$ & $2.38 \mathrm{E}-03$ & $\mathrm{M}_{26}$ & $1.35 \mathrm{E}-02$ \\
\hline $\mathrm{L}_{13}$ & $-8.10 \mathrm{E}-03$ & $\mathrm{~L}_{27}$ & $-2.89 \mathrm{E}-03$ & $\mathrm{M}_{13}$ & -0.15798473 & $\mathrm{M}_{27}$ & $3.16 \mathrm{E}-04$ \\
\hline
\end{tabular}


Table 10-The error rate values using the $6^{\mathrm{TH}}$ order polynomials equations ( $6 \mathrm{a} \& 6 \mathrm{~b}$ )

\begin{tabular}{|c|c|c|}
\hline Parameter & Error rate at X easting $(\mathrm{m})$ & Error rate at Y northing $(\mathrm{m})$ \\
\hline Maximum & 1.858521 & 0.057399 \\
\hline Minimum & 0.000134 & 0.000001 \\
\hline Average & 0.176834168 & 0.008532323 \\
\hline
\end{tabular}
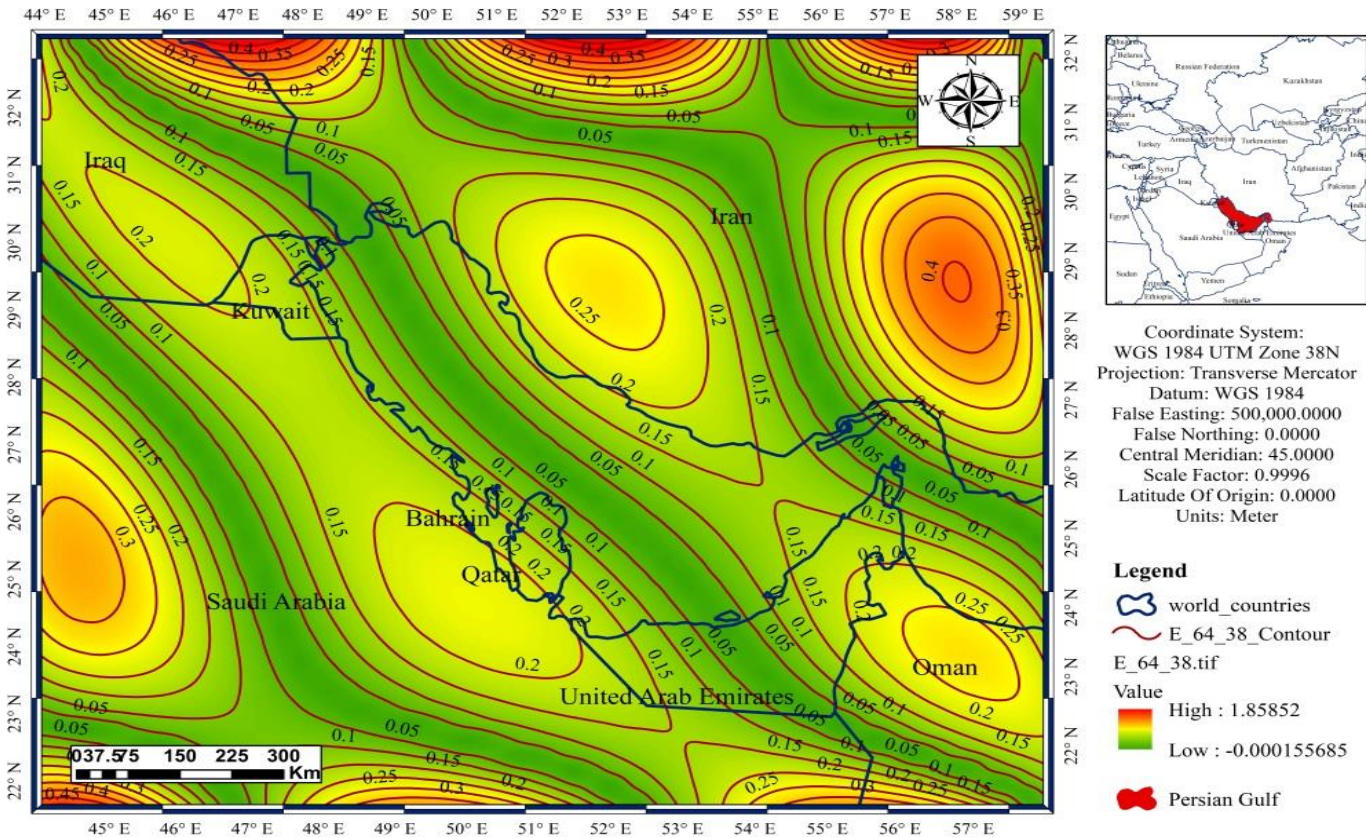

Coordinate System:
GS 1984 UTM Zone $38 \mathrm{~N}$ rojection: Transverse Mercator Datum: WGS 1984 False Easting: 500,000.000 False Northing: 0.0000 Central Meridian: 45.0000 Latitude Of Origin: 0.0000 Units: Meter

Legend

J world_countries

$\sim$ E 6438 Contour

E_64_38.tif

Value High : 1.85852

Low : -0.000155685

Figure 3- The distribution of error values at $\mathrm{x}$ easting using $6^{\text {th }}$ order polynomial.
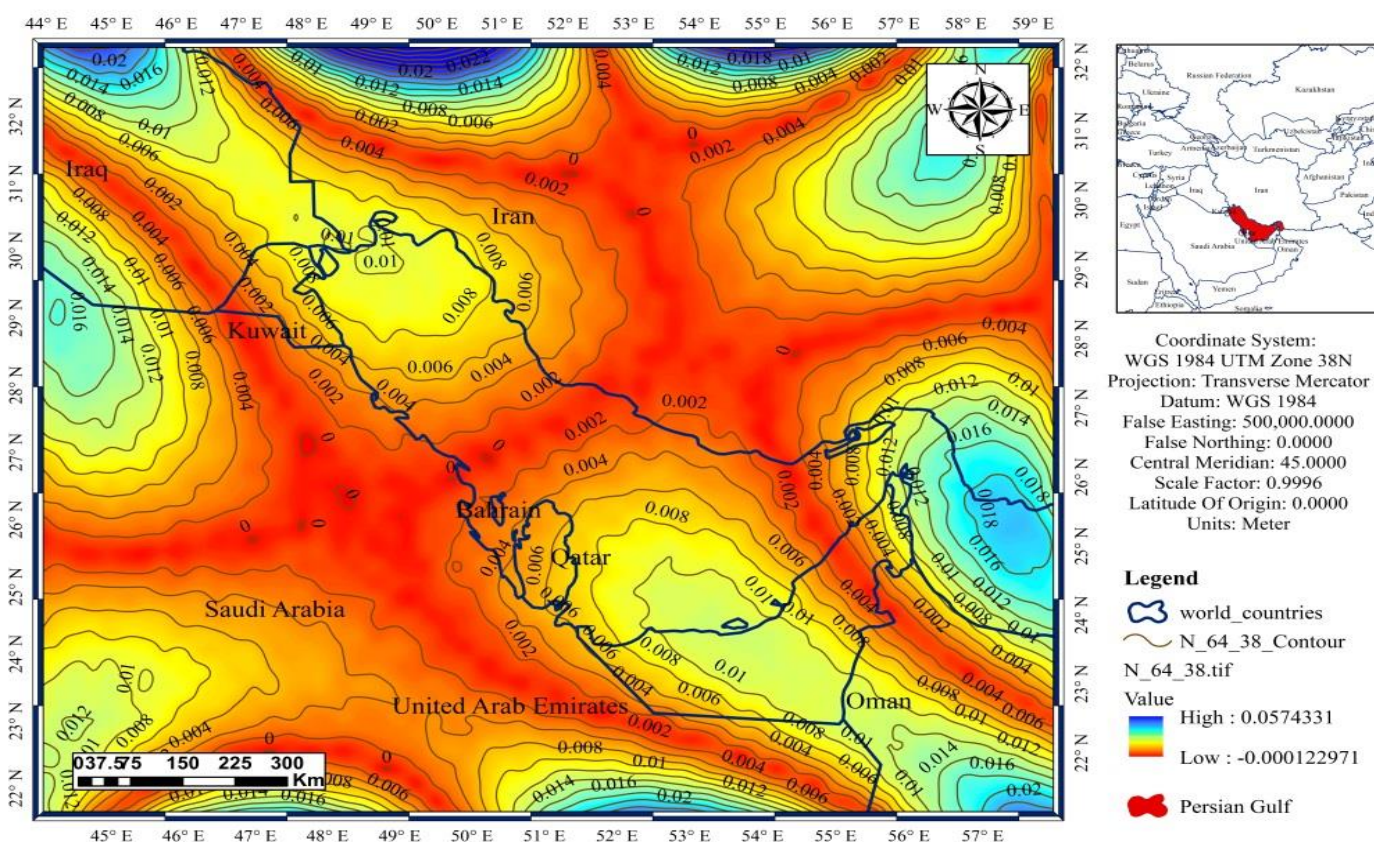

Figure 4- The distribution of error values at $Y$ northing using a $6^{\text {th }}$ order polynomial

\subsection{Backward}

Tables-11 and 12 present the values of coefficients and values of error rates at the studied longitudes and latitudes, using the $6^{\text {th }}$ order polynomials equations (9a and $9 \mathrm{~b}$ ) to test the accuracy of the converting process. Also, the error distributions are shown in Figures-5 and 6. 
Table 11- Backward values of parameters using the $6^{\text {th }}$ order polynomials equations ( $8 \mathrm{a} \& 8 \mathrm{~b}$ )

\begin{tabular}{|c|c|c|c|c|c|c|c|}
\hline Symbol & Coefficients & Symbol & Coefficients & Symbol & Coefficients & Symbol & Coefficients \\
\hline $\mathrm{O}_{0}$ & $1.82 \mathrm{E}-04$ & $\mathrm{O}_{14}$ & $1.20 \mathrm{E}-02$ & $\mathrm{P}_{0}$ & $3.68 \mathrm{E}-03$ & P14 & $-2.61 \mathrm{E}-03$ \\
\hline $\mathrm{O}_{1}$ & 0.215658 & $\mathrm{O}_{15}$ & $-1.35 \mathrm{E}-05$ & $\mathrm{P} 1$ & $-1.75 \mathrm{E}-02$ & P15 & $-1.63 \mathrm{E}-06$ \\
\hline $\mathrm{O}_{2}$ & $2.84 \mathrm{E}-02$ & $\mathrm{O}_{16}$ & $1.25 \mathrm{E}-03$ & $\mathrm{P} 2$ & 1.001526 & $\mathrm{P} 16$ & $-2.01 \mathrm{E}-04$ \\
\hline $\mathrm{O}_{3}$ & $-2.66 \mathrm{E}-03$ & $\mathrm{O}_{17}$ & $7.77 \mathrm{E}-04$ & $\mathrm{P} 3$ & $-1.58 \mathrm{E}-02$ & P17 & $1.15 \mathrm{E}-04$ \\
\hline $\mathrm{O}_{4}$ & $1.99 \mathrm{E}-02$ & $\mathrm{O}_{18}$ & $-4.66 \mathrm{E}-04$ & $\mathrm{P} 4$ & $-3.26 \mathrm{E}-03$ & P18 & $-3.43 \mathrm{E}-05$ \\
\hline $\mathrm{O}_{5}$ & $5.14 \mathrm{E}-02$ & $\mathrm{O}_{19}$ & $-1.09 \mathrm{E}-03$ & P5 & $-2.03 \mathrm{E}-02$ & P19 & $-2.41 \mathrm{E}-03$ \\
\hline $\mathrm{O}_{6}$ & $-1.62 \mathrm{E}-03$ & $\mathrm{O}_{20}$ & $5.70 \mathrm{E}-03$ & P6 & $4.99 \mathrm{E}-04$ & P20 & $-9.82 \mathrm{E}-04$ \\
\hline $\mathrm{O}_{7}$ & $6.84 \mathrm{E}-03$ & $\mathrm{O}_{21}$ & $-1.08 \mathrm{E}-05$ & P7 & $-1.15 \mathrm{E}-03$ & P21 & $3.59 \mathrm{E}-06$ \\
\hline $\mathrm{O}_{8}$ & $-2.21 \mathrm{E}-03$ & $\mathrm{O}_{22}$ & $5.42 \mathrm{E}-04$ & P8 & -0.01828 & P22 & $-1.82 \mathrm{E}-04$ \\
\hline $\mathrm{O}_{9}$ & $3.58 \mathrm{E}-02$ & $\mathrm{O}_{23}$ & $1.74 \mathrm{E}-04$ & P9 & $-4.71 \mathrm{E}-03$ & P23 & $-1.10 \mathrm{E}-04$ \\
\hline $\mathrm{O}_{10}$ & $4.71 \mathrm{E}-06$ & $\mathrm{O}_{24}$ & $8.52 \mathrm{E}-04$ & P10 & $2.26 \mathrm{E}-04$ & P24 & $-2.27 \mathrm{E}-04$ \\
\hline $\mathrm{O}_{11}$ & $3.34 \mathrm{E}-03$ & $\mathrm{O}_{25}$ & $-2.79 \mathrm{E}-04$ & P11 & $-1.18 \mathrm{E}-04$ & P25 & $7.13 \mathrm{E}-06$ \\
\hline $\mathrm{O}_{12}$ & $-6.93 \mathrm{E}-04$ & $\mathrm{O}_{26}$ & $-8.26 \mathrm{E}-04$ & P12 & $5.72 \mathrm{E}-04$ & P26 & $-8.68 \mathrm{E}-04$ \\
\hline $\mathrm{O}_{13}$ & $-2.15 \mathrm{E}-03$ & $\mathrm{O}_{27}$ & $2.13 \mathrm{E}-03$ & P13 & $-4.20 \mathrm{E}-03$ & P27 & $-4.38 \mathrm{E}-04$ \\
\hline
\end{tabular}

Table 12- The error rate values for the backward using the $6^{\text {th }}$ order polynomials equations $(8 \mathrm{a} \& 8 \mathrm{~b})$

\begin{tabular}{|c|c|c|}
\hline Parameter & Error rate at longitude (SEC) & Error rate at latitude (SEC) \\
\hline Maximum & $2.59 \mathrm{E}-05$ & $1.02 \mathrm{E}-05$ \\
\hline Minimum & $8.86 \mathrm{E}-10$ & $3.00 \mathrm{E}-10$ \\
\hline Average & $3.87 \mathrm{E}-06$ & $1.13 \mathrm{E}-06$ \\
\hline
\end{tabular}

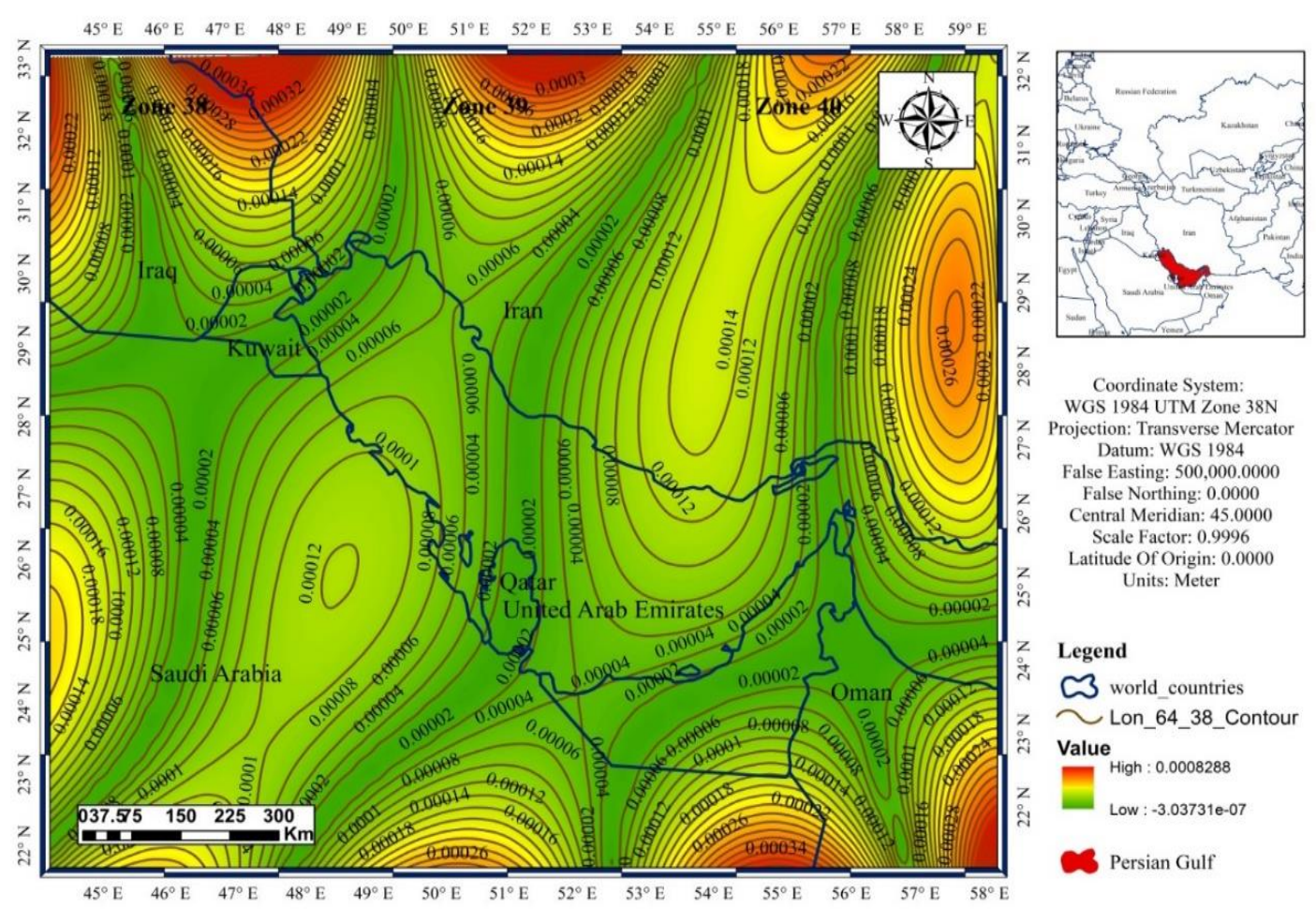

Figure 5- The distribution of error values at the longitudes using a $6^{\text {th }}$ order polynomial 

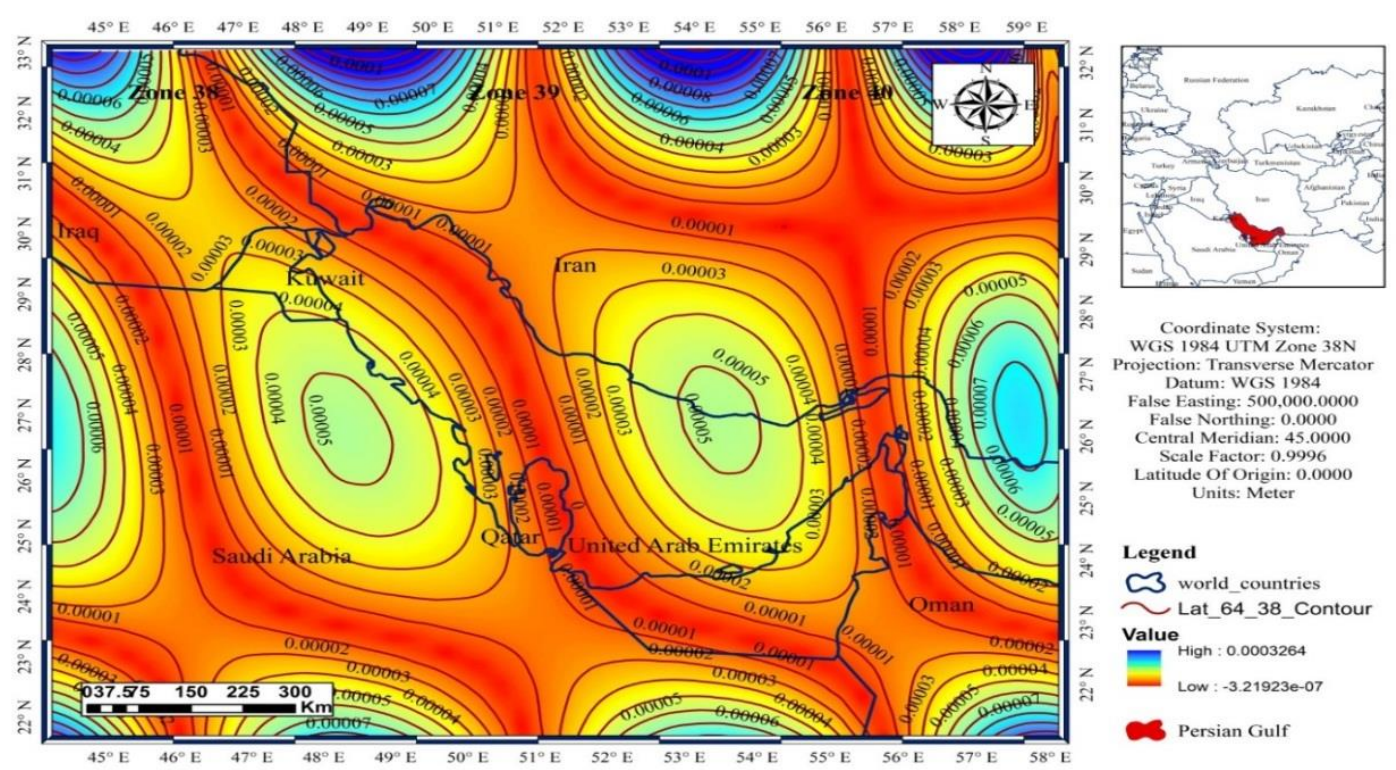

Figure 6- The distribution of error values at the latitudes using a $6^{\text {th }}$ order polynomial

\section{Conclusions}

1. This study showed that the application of the first test of the normalization method led to results that maintained a very high error rate, which resulted in the failure of the conversion process. However, when using the second test of the normalization method, the error rate was gradually reduced to the lowest ratio, when the sixth-degree polynomial equation was applied, which contributed to the success of the conversion and provided similar results to ideal conditions. Using the sixth-order polynomial equation, the value of the average error was approximately 0.17 meters at $X$ east and about 0.008 meters at $\mathrm{Y}$ north. These results show a very efficient model, on which we can rely for the transmission process of $\mathrm{X}$ east and $\mathrm{Y}$ north from (Longitude and Latitude) for the northern part of the Arabian Gulf, due to the high precision found in this model.

\section{References}

1. R. Brazeal, M. Sc, P. Eng, and P. Surv. 2016. "Issues with Extending a UTM Zone," pp. 1-14.

2. J. P. Snyder. 1997. Flattening the earth: two thousand years of map projections. University of Chicago Press.

3. A. Setiawan and E. Sediyono. 2018. "The use of google maps and universal transverse mercator (UTM) coordinate in land measurement of region in different zone," J. Theor. Appl. Inf. Technol., 96(23): 8071-8080.

4. A. State. 2014. A. Surveys, and R. State, "On the Conversion of Coordinates from Nigeria Transverse Mercator to Universal Transverse Mercator Using a Simple Mathematical Model," 4(4): 292-299.

5. C. F. F. Karney. 2011. "Transverse Mercator with an accuracy of a few nanometers," .

6. R. B. Langley and C. Chandler. "The UTM Grid System."

7. K. M. Kelly. 1989. "Coordinate Transformations, Universal Transverse Mercator/Geographic," Assoc. Ontario L. Surv.

8. J. W. Hager, J. F. Behensky, and B. W. Drew. 1989. "The Universal Grids: Universal Transverse Mercator (UTM) and Universal Polar Stereographic (UPS). Edition 1," Defense Mapping Agency Hydrographic/Topographic Center Washington Dc.

9. Sayed, B. H., \& George, L. E. 2020. Flat Model for Representing Contiguous UTM Coordinates over Iraq Territory. Iraqi Journal of Science, 908-919.

10. BEYER , W . H .1987. " CRC Standard Mathematical Tables ", 1987 , CRC PRESS , pages : 212," p. 56839809.

11. U. C. Merzbach and C. B. Boyer. 2011. A history of mathematics. John Wiley \& Sons, 2011.

12. B. Taylor. 1969. "Methodus Incrementorum Directa et Inversa [Direct and Reverse Methods of Incrementation]," Pearsonianis prostant apud Gul. Innys, pp. 21-23, 1969.

13. Behadili, S. F., \& Sayed, B. H. 2019. Oli and Gas Explorations via Satellite Remote Sensing Techniques for AL_Nasiriya. Iraqi Journal of Science, 2308-2314. 